\title{
Evaluating GAIA performances on eclipsing binaries
}

\section{Orbits and stellar parameters for V505 Persei, V570 Persei and OO Pegasi}

\author{
U. Munari ${ }^{1,2}$, T. Tomov ${ }^{1,3}$, T. Zwitter ${ }^{4}$, E. F. Milone ${ }^{5}$, J. Kallrath ${ }^{6,7}$, P. M. Marrese ${ }^{1,2}$, F. Boschi ${ }^{1,8}$, \\ A. Prša ${ }^{4}$, L. Tomasella ${ }^{1}$, and D. Moro ${ }^{9}$ \\ 1 Osservatorio Astronomico di Padova, Sede di Asiago, 36012 Asiago (VI), Italy \\ 2 CISAS, Centro Interdipartimentale Studi ed Attività Spaziali dell'Università di Padova, Italy \\ 3 Centre for Astronomy, Nicholaus Copernicus University, ul. Gagarina 11, 87-100 Torun, Poland \\ 4 University of Ljubljana, Department of Physics, Jadranska 19, 1000 Ljubljana, Slovenia \\ 5 Physics and Astronomy Department, University of Calgary, Calgary T2N 1N4, Canada \\ 6 BASF-AG, ZDP/C-C13, D-67056 Ludwigshafen, Germany \\ 7 Astronomy Department, University of Florida, Gainesville, FL 32611, USA \\ 8 Dipartimento di Fisica dell'Università di Milano, via Celoria 20, 20131 Milano, Italy \\ ${ }^{9}$ Dipartimento di Astronomia dell'Università di Padova, Osservatorio Astrofisico, 36012 Asiago (VI), Italy
}

Received 13 April 2001 / Accepted 28 August 2001

\begin{abstract}
The orbits and physical parameters of three detached, double-lined A-F eclipsing binaries have been derived combining $H_{\mathrm{P}}, V_{\mathrm{T}}, B_{\mathrm{T}}$ photometry from the Hipparcos/Tycho mission with 8500-8750 $\AA$ ground-based spectroscopy, mimicking the photometric+spectroscopic observations that should be obtained by GAIA, the approved Cornerstone 6 mission by ESA. This study has two main objectives, namely $(a)$ to derive reasonable orbits for a number of new eclipsing binaries and $(b)$ to evaluate the expected performances by GAIA on eclipsing binaries and the accuracy achievable on the determination of fundamental stellar parameters like masses and radii. It is shown that a $1 \%$ precision in the basic stellar parameters can be achieved by GAIA on well observed detached eclipsing binaries provided that the spectroscopic observations are performed at high enough resolution. Other types of eclipsing binaries (including semi-detached and contact types) and different spectral types will be investigated in subseguent papers in this series.
\end{abstract}

Key words. surveys - stars: fundamental parameters - binaries: eclipsing - binaries: spectroscopic

\section{Introduction}

Eclipsing binaries are a prime tool to derive fundamental stellar parameters like mass and radius, or the temperature scale. The study of eclipsing binaries is by no means a simple task as evidenced by the fact that stellar parameters have been derived with an accuracy of $1 \%$ or better for fewer than a hundred objects. The prospects for the future seen however quite bright given the expected performances of the GAIA mission.

GAIA has just been selected as the next ESA Cornerstone 6 mission and it is designed to obtain extremely precise astrometry (in the micro-arcsec regime), multi-band photometry and medium/high resolution spectroscopy for a large sample of stars. The goals as depicted in the mission Concept and Technology Study (ESA SP2000-4) call for astrometry and broad band photometry

Send offprint requests to: U. Munari, e-mail: munari@astras.pd.astro.it to be collected for all stars down to $V \sim 20$ mag over the entire sky $\left(\sim 1 \times 10^{9}\right.$ stars $)$, with brighter magnitude limits for spectroscopy and intermediate band photometry. Each target star should be measured around a hundred times during the five year mission life-time, in a fashion similar to the highly successful Hipparcos operational mode. The astrophysical guidelines of the GAIA mission are discussed by Gilmore et al. (1998) and Perryman et al. (2001), an overview of the GAIA payload and spacecraft is presented by Mérat et al. (1999), while the goals of GAIA spectroscopy and photometry are discussed by Munari (1999a,b).

With photometry complete down to $V=20$ mag and spectroscopy down to $V=15$ mag or so (depending on the final optical design and overall throughput), one may expect to detect a large number of eclipsing binaries. Let's roughly estimate how much. The number of stars brighter than $V=15$ (thus bright enough for GAIA to obtain both photometry and spectroscopy) is $5 \times 10^{7}$ and their average 
Table 1. Program eclipsing binaries. Data from the Hipparcos Catalogue. $H_{\mathrm{P}}, B_{\mathrm{T}}, V_{\mathrm{T}}$ are median values (i.e. out-of-eclipse).

\begin{tabular}{lllllllll}
\hline Name & Spct. & $H_{\mathrm{P}}$ & $B_{\mathrm{T}}$ & $V_{\mathrm{T}}$ & $\begin{array}{c}\alpha_{\mathrm{J} 2000} \\
(\mathrm{~h} \mathrm{~m} \mathrm{~s})\end{array}$ & $\begin{array}{c}\delta_{\mathrm{J} 2000} \\
\left({ }^{\circ} / \prime\right)\end{array}$ & $\begin{array}{c}\text { parallax } \\
(\operatorname{mas})\end{array}$ & $\begin{array}{c}\mu_{\alpha} \\
\left(\operatorname{mas}^{-1}\right)\end{array}$ \\
\hline
\end{tabular}

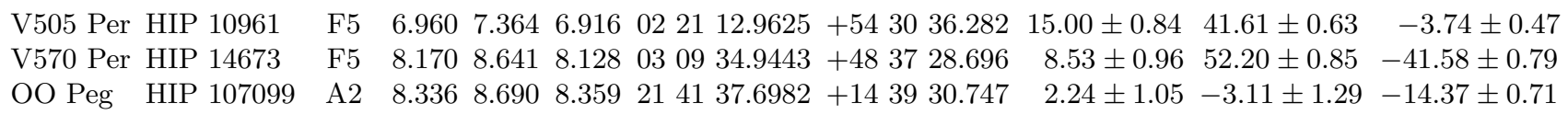

spectral type is about G7. Scaling the Hipparcos results (993 detected among the 118218 stars surveyed or $0.8 \%$ of the total, Oblak \& Kurpinska-Winiarska 2000), $\sim 4 \times 10^{5}$ of them would be eclipsing binaries. At an average G7 spectral type it may be estimated that about $25 \%$ of them $\left(\sim 1 \times 10^{5}\right)$ will be double-lined in GAIA spectral observations (cf. Carquillat et al. 1982). Even if for only $1 \%$ of them it should be possible to derive orbits and stellar parameters at $1 \%$ precision, this still would be $\sim 25 \times$ more have than so far been collected from devoted ground-based observing campaigns during the last century (cf. Andersen 1991).

GAIA is baselined to rotate every three hours around an axis (pointing $55^{\circ}$ away from the Sun) that completes a precession cycle every 76 days. Stars will be recorded while they transit across the field of view of the three fixed telescopes on board (separated by roughly $120^{\circ}$ ), which use as detectors mosaics of CCDs operating in time-delayed integration mode. Thus, how many times and when a star will be observed depends on its position on the sky and the GAIA scanning law. Along the $\sim 3$ hour spin period a given star will pass in turn every hour in front of one of the GAIA's FoV. Because the currently baselined $~ 15$ photometric bands (between broad and narrow, counting also duplications among different FoV) will be distributed among the three FoV, over one spin period there will be three groups of photometric measurements (even if not all in the same bands). Spectra will be collected in only one FoV (covering the region $8500-8750 \AA$ centered on the near infrared Ca II triplet and the head of the Paschen series). On average, over the GAIA 5-year lifetime a given star will be observed over 67 spin rotations. Thus, on average 67 spectra and $1005=67 \times 15$ photometric observations will be collected, the latter being however grouped into only $201=67 \times 3$ independent epochs.

Table 2. Number of Hipparcos $\left(H_{\mathrm{P}}\right)$ and Tycho $\left(B_{\mathrm{T}}, V_{\mathrm{T}}\right)$ photometric data and ground based radial velocity observations, their mean $S / N$ and standard error for the three program stars.

\begin{tabular}{|c|c|c|c|c|c|c|c|c|}
\hline & \multicolumn{2}{|r|}{ Hip } & \multicolumn{3}{|c|}{ Tyc } & \multicolumn{3}{|c|}{$R V$} \\
\hline & $N$ & $\sigma\left(H_{\mathrm{P}}\right)$ & $N$ & $\sigma\left(B_{\mathrm{T}}\right)$ & $\sigma\left(V_{\mathrm{T}}\right)$ & $N$ & $S / N$ & $\sigma(R V)$ \\
\hline OO Peg & 73 & 0.013 & 61 & 0.110 & 0.109 & 21 & 38 & 3.5 \\
\hline V570 Per & 92 & 0.014 & 120 & 0.111 & 0.100 & 28 & 57 & 3.6 \\
\hline V505 Per & 122 & 0.009 & 152 & 0.057 & 0.051 & 20 & 91 & 1.7 \\
\hline
\end{tabular}

How does this sampling by GAIA compare with examples of state-of-the-art ground-based studies of eclipsing binary stars? Generally, between 30 and 60 good spectra (secured away from conjunction phases) are enough to characterize well the radial velocity curve and the spectroscopic orbit, while many hundreds to several thousand photometric points are necessary to cover in detail the photometric lightcurve (and the eclipse phases in particular) and to derive an accurate photometric solution. The 67 spectra per star secured on average by GAIA should put the spectral monitoring of eclipsing binaries on the safe side even for the faintest recorded systems. Where GAIA could fall short of optimal coverage is with the photometric observations. A pertinent example is given by Hipparcos, which scanned the sky in a fashion very similar to GAIA: with a mean of 110 observations per star, the eclipses of many binaries have been covered by less than 10 photometric points (for example see the case of V505 Per in Fig. 3 below).

The limit on the number of photometric observations is intrinsic to the GAIA operation mode and cannot be changed by large margins. It is also quite evident that a devoted effort from an international consortium to perform follow-up observations of even a small subsample of the $\sim 4 \times 10^{5}$ eclipsing binaries detected by GAIA would be an unrealistic goal. Moreover, it can be anticipated that such follow-up projects could take many years to be completed and could obviously begin only after the end of the GAIA mission and the completion of the data reduction and dissemination phase. It thus seems quite relevant to investigate the accuracy to which eclipsing binaries can be investigated on the base of the GAIA data alone. The present

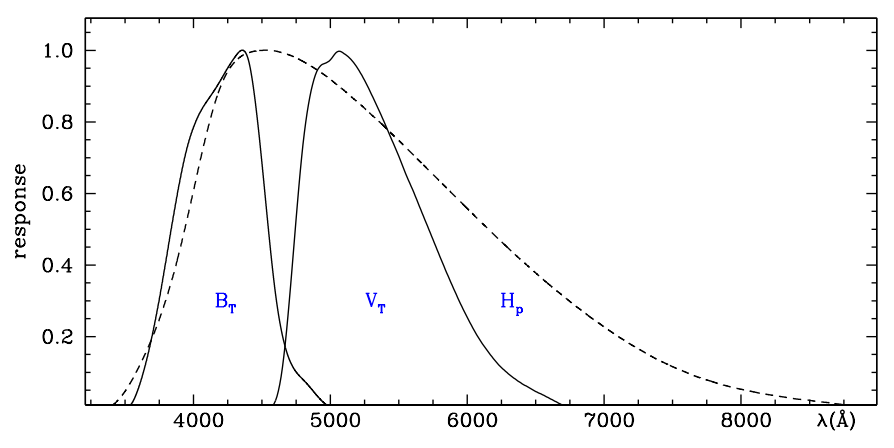

Fig. 1. Transmission of the Hipparcos $H_{\mathrm{P}}$ and Tycho $B_{\mathrm{T}}, V_{\mathrm{T}}$ bands. 
Table 3. Journal of radial velocity data. The columns give the spectrum number (as from the Asiago $1.82 \mathrm{~m} \mathrm{Echelle+CCD} \mathrm{log}$ book), the heliocentric JD (+2451000), the radial velocities and their standard errors for both components. An asterisk marks the spectra with too severe blending of the lines from both components for meaningful separate measurement. The latter have not been used in modeling the binaries.

\begin{tabular}{|c|c|c|c|c|c|c|c|c|c|c|c|c|c|c|c|c|c|}
\hline \multicolumn{6}{|c|}{ V505 Per } & \multicolumn{6}{|c|}{ V570 Per } & \multicolumn{6}{|c|}{ OO Peg } \\
\hline \# & HJD & $\mathrm{RV}_{1}$ & $\operatorname{err}_{1}$ & $\mathrm{RV}_{2}$ & $\mathrm{err}_{2}$ & $\#$ & HJD & $\mathrm{RV}_{1}$ & $\operatorname{err}_{1}$ & $\mathrm{RV}_{2}$ & $\mathrm{err}_{2}$ & $\#$ & HJD & $\mathrm{RV}_{1}$ & $\operatorname{err}_{1}$ & $\mathrm{RV}_{2}$ & $\mathrm{err}_{2}$ \\
\hline 30349 & 147.4711 & 79.1 & 2.1 & -81.5 & 1.5 & 30364 & 147.5377 & -78.1 & 4.2 & 116.1 & 3.9 & 30230 & 126.2919 & -91.6 & 4.1 & 119.9 & 1.4 \\
\hline 30721 & 153.4598 & -91.7 & 0.5 & 89.7 & 3.3 & 30716 & 153.4343 & -91.8 & 1.0 & 135.3 & 4.5 & 30286 & 147.2247 & -100.4 & 3.8 & 121.4 & 4.6 \\
\hline $30785 *$ & 154.4885 & -0.7 & 1.6 & -0.7 & 1.6 & 30796 & 154.5488 & 109.8 & 5.4 & -61.5 & 3.9 & 30685 & 153.2106 & -106.6 & 3.3 & 117.8 & 2.8 \\
\hline 30828 & 155.3391 & 81.5 & 2.6 & -85.3 & 2.2 & 30842 & 155.4289 & -88.9 & 2.1 & 123.7 & 1.3 & 30745 & 154.1937 & 53.6 & 3.5 & -38.8 & 3.1 \\
\hline 30894 & 156.3391 & 42.5 & 1.8 & -46.0 & 0.3 & 30903 & 156.4605 & 107.4 & 2.9 & -65.9 & 1.1 & 30815 & 155.1945 & 69.8 & 3.3 & -54.9 & 4.4 \\
\hline 31155 & 165.3867 & -38.0 & 0.9 & 40.7 & 0.6 & 31161 & 165.4133 & 68.6 & 4.7 & -22.6 & 2.3 & 30874 & 156.2212 & -101.3 & 2.3 & 118.3 & 7.8 \\
\hline 31214 & 166.4223 & -82.1 & 0.8 & 82.4 & 1.4 & 31219 & 166.4476 & -53.1 & 4.3 & 95.1 & 1.9 & 31117 & 165.2149 & -101.3 & 1.1 & 119.4 & 2.6 \\
\hline 31263 & 167.3923 & 22.0 & 0.1 & -22.8 & 0.9 & 31268 & 167.4216 & 110.9 & 6.7 & -62.3 & 2.0 & 31187 & 166.1952 & 66.5 & 2.2 & -59.2 & 3.7 \\
\hline 31312 & 169.4268 & -12.4 & 1.8 & 14.2 & 1.1 & 31317 & 169.4937 & 138.3 & 4.4 & -88.1 & 2.3 & 31243 & 167.2751 & 40.9 & 3.8 & -36.4 & 4.9 \\
\hline 31453 & 197.4223 & 73.6 & 1.6 & -74.1 & 1.8 & 31456 & 197.4483 & -14.6 & 5.2 & 71.3 & 2.1 & 31431 & 197.1985 & 36.1 & 0.8 & -1.7 & 9.1 \\
\hline 31455 & 197.4386 & 73.7 & 1.2 & -79.1 & 1.9 & 31779 & 206.3250 & -45.5 & 3.5 & 87.6 & 1.8 & 31621 & 201.2230 & -94.6 & 5.7 & 109.4 & 1.9 \\
\hline 31817 & 209.2477 & -22.9 & 2.4 & 19.8 & 3.6 & 31821 & 209.3057 & 126.4 & 4.9 & -76.1 & 1.8 & 33109 & 400.5219 & -55.4 & 1.6 & 73.4 & 1.7 \\
\hline 31941 & 216.3976 & -72.8 & 2.0 & 74.4 & 2.9 & 31967 & 217.4277 & 66.5 & 3.4 & -19.5 & 1.3 & 33313 & 481.3637 & -100.2 & 9.9 & 95.3 & 9.9 \\
\hline 33294 & 480.5084 & 86.8 & 2.1 & -83.1 & 1.0 & 32056 & 224.4361 & 103.4 & 6.2 & -61.3 & 2.1 & $33400 *$ & 506.2928 & 9.7 & 1.8 & 9.7 & 1.8 \\
\hline 33296 & 480.5286 & 85.5 & 2.5 & -87.6 & 0.8 & 32080 & 225.3604 & -60.6 & 7.1 & 90.4 & 4.5 & 33437 & 507.3198 & 82.7 & 1.9 & -67.6 & 1.7 \\
\hline 33325 & 485.4974 & 59.8 & 0.5 & -59.9 & 0.5 & 33297 & 480.5490 & -72.5 & 2.9 & 112.4 & 6.5 & 33467 & 508.1901 & -83.2 & 4.9 & 109.5 & 3.5 \\
\hline 33376 & 505.4180 & 54.3 & 2.2 & -59.8 & 1.2 & $33328 *$ & 485.5251 & 14.8 & 2.9 & 14.8 & 2.9 & $33512 *$ & 509.1837 & 6.6 & 2.0 & 6.6 & 2.0 \\
\hline 33674 & 531.3520 & 89.5 & 3.2 & -90.8 & 0.5 & $33378 *$ & 505.4516 & 30.5 & 2.8 & 30.5 & 2.8 & 33696 & 533.3212 & 66.6 & 3.8 & -45.0 & 0.5 \\
\hline 33988 & 565.3701 & 80.7 & 3.1 & -87.3 & 1.2 & $33410 *$ & 506.4728 & 19.8 & 2.2 & 19.8 & 2.2 & 35244 & 714.5465 & -99.2 & 2.5 & 107.7 & 6.5 \\
\hline \multirow[t]{9}{*}{34026} & 567.2156 & -89.5 & 1.9 & 92.9 & 4.7 & 33431 & 507.2567 & -45.4 & 2.0 & 87.9 & 2.1 & 35745 & 798.3182 & -65.1 & 1.8 & 85.1 & 0.6 \\
\hline & & & & & & $33486 *$ & 508.3856 & 20.9 & 0.4 & 20.9 & 0.4 & 35783 & 799.4859 & 116.8 & 3.6 & -105.9 & 6.8 \\
\hline & & & & & & $33528 *$ & 509.3303 & 23.0 & 2.8 & 23.0 & 2.8 & & & & & & \\
\hline & & & & & & 33706 & 533.4862 & -94.8 & 3.2 & 131.2 & 2.9 & & & & & & \\
\hline & & & & & & 33868 & 561.2317 & 134.0 & 6.1 & -76.1 & 4.2 & & & & & & \\
\hline & & & & & & 33870 & 561.3084 & 123.3 & 7.5 & -74.6 & 1.5 & & & & & & \\
\hline & & & & & & 33932 & 564.2202 & -62.8 & 4.3 & 97.8 & 2.1 & & & & & & \\
\hline & & & & & & $33965 *$ & 564.4945 & 18.6 & 8.1 & 18.6 & 8.1 & & & & & & \\
\hline & & & & & & 34066 & 570.2546 & 49.8 & 6.6 & -1.9 & 6.1 & & & & & & \\
\hline
\end{tabular}

series of papers is devoted to precisely this aim, combining ground based spectroscopy over the 8500-8750 A interval chosen for GAIA with existing Hipparcos/Tycho photometry that well mimics the GAIA photometric harvest.

To be precise, GAIA is expected to collect a number of photometric points per star, per band similar to Hipparcos. However Hipparcos collected data in just three bands $\left(H_{\mathrm{P}}, B_{\mathrm{T}}, V_{\mathrm{T}}\right.$, see Fig. 1$)$, while GAIA is baselined to operate $\sim 10$ bands ( 5 broad in two FoV and 5 intermediate in the third FoV). Therefore, combining all data in all bands, GAIA should provide $\sim 3 \times$ more photometric points per star and distribute them over $\sim 2 \times$ independent epochs compared to Hipparcos.

The dispersion currently baselined for GAIA spectroscopy is $0.75 \AA /$ pix. However, a different dispersion could eventually be selected between the $0.25-1.5 \AA /$ pix boundaries investigated during the mission planning phase. We decided to carry out our observations at the highest resolution considered possible for GAIA (0.25 $\AA /$ pix), for two basic reasons: $(a)$ to evaluate the maximum GAIA potential, and $(b)$ because data secured at a higher resolution can always be degraded to mimic lower resolution observations, while the contrary is obviously not feasible.

Finally, it is worth noticing that GAIA will be able to follow the astrometric motion around the baricenter of the components of binary stars separated by at least 0.05 arcsec, thus providing a completely independent method to derive fundamental stellar parameters for those binaries in the solar neighbourhood whose orbital period is not much longer than the GAIA mission lifetime.

\section{Target selection}

At the relatively bright magnitude limit reached by Tycho observations (limit of completeness $V \sim 10.5$ ) the average color of the stars corresponds to a G0 spectral type. At the fainter magnitudes reached by GAIA the stars will be increasingly redder, with an average K0 spectral type at $V=15 \mathrm{mag}$. Therefore the target stars for the present series of papers will be biased toward G-K spectral types, with fewer target stars among the A, F and M types.

All targets in this paper series are selected among the eclipsing binaries observed by the Hipparcos/Tycho 
mission, with preference to those lacking an orbital solution in the literature.

In this first paper we investigate the three $\mathrm{A}-\mathrm{F}$, detached eclipsing binaries listed in Table 1 . The number of available photometric and spectroscopic data and their mean standard errors are given in Table 2. V505 Per has an accurate spectroscopic/photometric solution from extensive ground observations (Marshall et al. 1997) and serves for comparison, while for V570 Per and OO Peg the orbit is derived here for the first time.

In following papers in this series we will also consider semi-detached and contact eclipsing binaries as well as eclipsing binaries with one or both of the components being itself a variable star.

\section{Radial velocities}

A $0.25 \AA /$ pix dispersion and a $\sim 0.50 \AA$ resolution over the 8500-8750 $\AA$ wavelength range (therefore a resolving power $R=\lambda / \Delta \lambda=17000$ ) will be maintained throughout this paper series.

The spectroscopic observations have been obtained with the Echelle+CCD spectrograph on the $1.82 \mathrm{~m}$ telescope operated by Osservatorio Astronomico di Padova atop Mt. Ekar (Asiago). A 2.2 arcsec slit width was adopted to match the $R=\lambda / \Delta \lambda=17000$ requirement. The detector was a UV coated Thompson CCD $1024 \times 1024$ pixel, 19 micron square size. The GAIA spectral range is covered without gaps in a single order by the Asiago Echelle spectrograph. The actual observations however extended over a much larger wavelength interval (4500-9000 $\AA$ ). Here we will limit the analysis to the GAIA spectral interval; the remaining, much larger wavelength domain will be analyzed elsewhere together with devoted multi band photometry from pointed ground based observations.

A sub-sample of the spectra collected at representative phases is presented in Fig. 2 for the three program stars.

The spectra have been extracted and calibrated in a standard fashion using the IRAF software package running on a PC under the Linux operating system. The stability of the wavelength scale of the Asiago Echelle spectrograph has been discussed in detail by Munari \& Lattanzi (1992) and Munari \& Tomasella (1999a). We checked it on each recorded spectrum by measuring the wavelengths of several telluric absorption lines that are abundant in the Echelle orders next to the one covering the GAIA spectral window (cf. Munari 1999b). No wavelength shift has been detected in excess of $0.2 \mathrm{~km} \mathrm{~s}^{-1}$ which nicely compares with the intrinsic precision limit of radial velocities from cross-correlation techniques $\left( \pm 0.01\right.$ pixels $=0.1 \mathrm{~km} \mathrm{~s}^{-1}$ for the chosen instrumental set-up).

The radial velocities on the spectra of the three targets have been measured both in a line-by-line fashion as well as via cross-correlation.

In the line-by-line approach we considered for all the program binaries only the three CaII lines (8498.018,

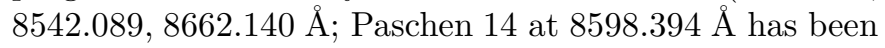

used too, but only on the best OO Peg spectra). To measure the line wavelengths we tested different approaches: profile fitting (Gaussian, Lorentzian or voigt profiles), bisector method and line photo-centering (center1d tool in IRAF). They gave nearly equivalent results, with the line-centering performing slightly better on average.

The superior intrinsic potential of the cross-correlation approach (fxcor and xcsao tools in IRAF) is generally only marginally exploited in real, ground-based spectra because of the need for a normalization of the continuum spectrum before running the cross-correlation. The normalization is particularly critical for our spectra (A and F spectral types, cf. Table 1) because they are dominated by a few broad lines with extended wings and nearly lineempty continuum in between. The shape of the continuum is badly affected by the blaze function (particularly peaked in Echelle low-number and near-IR orders) and by less predictable effects arising at the spectrograph slit and condensations on the dewar window. As templates for the cross-correlation we used both single-lined spectra at conjunctions, as well as real and synthetic spectra from the Munari \& Tomasella (1999b), Munari \& Castelli (2000) and Castelli \& Munari (2001) databases.

The radial velocities that we have measured are listed in Table 3. They are those obtained via cross-correlation. Manual measurements of individual lines has provided similar results, only less accurate.

\section{Hipparcos/Tycho photometry}

The input photometric data for the three program stars are those provided by the Hipparcos/Tycho mission. Those for V505 Per and OO Peg have been taken directly from the mission final databases on CD-ROMs, while for V570 Per the data (which were not previously published/distributed given their poorer quality) were obtained upon request from ESA.

The Hipparcos/Tycho mission collected data in three photometric bands (cf. Fig. 1). The Hipparcos $H_{\mathrm{P}}$ band is the most accurate of the three (cf. Table 2) and resembles white light measurements. The Tycho $B_{\mathrm{T}}$ and $V_{\mathrm{T}}$ bands are similar to the Johnson equivalent bands, as indicated by the transformation equation provided in the explanatory notes of the Hipparcos/Tycho Catalogue:

$$
\begin{aligned}
V_{\mathrm{J}} & =V_{\mathrm{T}}-0.090 \times(B-V)_{\mathrm{T}} \\
(B-V)_{\mathrm{J}} & =0.850 \times(B-V)_{\mathrm{T}} .
\end{aligned}
$$

The photometric bands of the Hipparcos/Tycho mission have been further discussed by Bessell (2000).

\section{Modeling}

The WD98 code used for the analyses is the successor program to WD95 described by Kallrath et al. (1998), the philosophy behind which was described in Kallrath \& Milone (1999). WD98 includes the version of the WilsonDevinney code distributed by Wilson (1998), namely the 


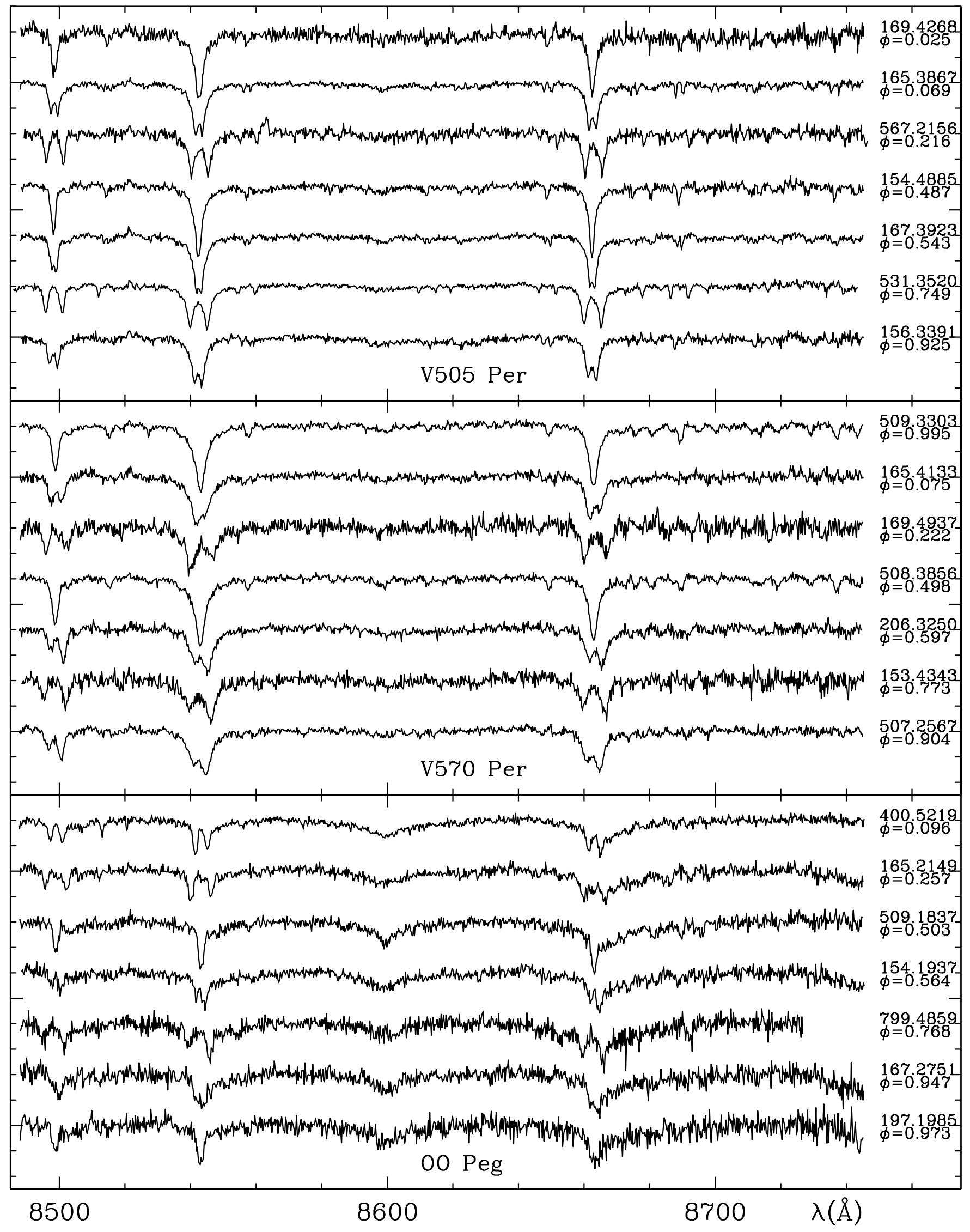

Fig. 2. A sample of the recorded spectra for the program stars. The HJD (+2451000) and the orbital phase (according to orbital elements listed in Table 4) are given on the right. 
options of having (a) time rather than phase as input data, (b) a square root limb-darkening law (in addition to the linear and logarithmic options of WD95), (c) atmospheric scattering effects in modeling the light curve, $(d)$ adjustment of the period and epoch within the WilsonDevinney code, and $(e)$ adjustment of a linear change in period and in the argument of periastron. WD98 permits iterative operation, and a variable damping factor as the solution is approached, to minimize the effects of correlations among the parameters, and retains Kurucz atmosphere models for the stellar components. We use logarithmic limb-darkening coefficients for the passbands computed from Kurucz models by W. VanHamme, and the desktop interpolation program of D. Terrell (private communications to EFM).

For all three stars investigated in this study the following steps were taken. The photometric data were plotted and the mean of the maxima used to normalize the light of the system. The initial epoch and period were taken from the Hipparcos solution (listed in the Hipparcos Catalogue) or from Marschall et al. (1997) for V505 Per, the temperature of the hotter star from the spectral classification, the initial temperature of the cooler star from the ratio of the depths of the minima, initial potentials set arbitrarily so that the stars did not exceed their critical surfaces (in modeling contact and over-contact systems, this procedure is changed, of course). Initially, all runs were carried out with linear limb-darkening and single-pass reflection treatment. The $H_{\mathrm{P}}, V_{\mathrm{T}}, B_{\mathrm{T}}$ photometric data and the radial velocity curves for both components were modeled together. The passband-specific flux ratio files (containing the ratio of the atmosphere to blackbody fluxes) were created from Kurucz atmosphere models by C. R. Stagg (private communication to EFM).

The parameters that were adjusted initially were the semi-major axis $(a)$, baricentric radial velocity $\left(V_{\gamma}\right)$, inclination $(i)$, temperature of star $2\left(T_{2}\right)$, modified Kopal potentials $\left(\Omega_{1,2}\right)$, mass ratio $\left(q=m_{2} / m_{1}\right)$, epoch, period $(P)$, and relative luminosity in each passband of star 1 $\left(L_{1_{\lambda}}\right)$, where star 2 is the component closer to us at the part of the light curve designated as phase 0.0.

After the computer-generated corrections for the epoch and period fell below their standard errors by at least $50 \%$, these parameters were no longer adjusted in subsequent runs, to improve the precision of the remaining parameters. Note that the potentials of both stars were permitted to vary (thus the stellar sizes are constrained by the eclipses alone).

\section{Results}

For all program stars the solutions agreed well inside the uncertainties with null eccentricity. We then fixed $e=0.0$ and reran the solutions.

As the initial temperature for the primary (hotter) star we adopted the one corresponding to the spectral type (from Table 1) according to Popper (1980). No reddening correction was adopted for these nearby binary systems (missing also accurate spectral classifications of both components).

For all the program stars there is no discernible sign of departure from spherical symmetry for either component: $r_{\text {pole }}=r_{\text {point }}=r_{\text {side }}=r_{\text {back }}$. The program stars are therefore examples of well-detached eclipsing systems.

The goal of this paper is to evaluate the potentials of GAIA observations on eclipsing binaries and not to discuss in detail the solution obtained for each program star. To this end we will refrain from listing and discussing unnecessary minor details about the modeling solutions. Moreover, we used all available photometric data with the aim of simulating automatic treatment of GAIA data; it is however clear from Figs. 3-5 that using only $H_{\mathrm{P}}$ data (ignoring $B_{\mathrm{T}}$ and $V_{\mathrm{T}}$ ones) would have reduced the errors on some of the stellar parameters in Table 4, in particular the radii and the derived quantities (like bolometric magnitudes and surface gravities). Therefore, the software that will actually deal with GAIA data should be smart enough to exclude from the analysis the whole lightcurves that would only add noise to the solution.

\subsection{V505 Persei}

The derived parameters for V505 Per are listed in Table 4, where they are compared to the Marshall et al. (1997) solution which should be correct to about $1 \%$ or better, and thus serves as the "true, intrinsic" set of values to compare with. The temperature from spectral type for the primary star would be $6460 \mathrm{~K}$. We adopted $6430 \mathrm{~K}$ derived from the color index $b-y=+0.287$ given by Hauck \& Mermilliod (1998) and the Popper (1980) calibration of $b-y$ into temperature.

Marshall et al. used 63 spectra secured at $R=\lambda / \Delta \lambda=$ 43000 compared to our only 20 at $R=\lambda / \Delta \lambda=17000$. This of course reflects an intrinsically higher precision of each observation, a firmer radial velocity orbital solution and a lower scatter of points along the computed curve for the Marshall et al. work. Nevertheless, determinations of individual masses and semi-major axis coincide inside the errors.

The surface temperatures of the two components are constrained to $\pm 60 \mathrm{~K}$ using the Hipparcos/Tycho threeband photometry. GAIA will obtain photometric data on $\sim 11$ bands, carefully placed and shaped to enhance the sensitivity, diagnostic capabilities and disentangling abilities for temperature, luminosity, metallicity and gravity overlapping effects. It is therefore quite safe to assume that the error on the temperature estimate that GAIA could achieve on eclipsing binaries similar to V505 Per will be significantly better than the already good $\pm 60 \mathrm{~K}$ allowed by Hipparcos/Tycho data.

The other parameters are satisfactorily determined, usually coincident inside the reported formal errors with the Marshall et al. solution.

The largest discrepancy between our GAIA-like solution and the Marshall et al. work is - of course - in the 
Table 4. Modeling solutions. The uncertainties are formal mean standard errors to the solution. The last four rows gives the standard deviation of the observed points from the derived orbital solution.

\begin{tabular}{|c|c|c|c|c|}
\hline \multirow[t]{2}{*}{ parameter (units) } & \multirow[t]{2}{*}{ V570 Per } & \multirow[t]{2}{*}{ OO Peg } & \multicolumn{2}{|c|}{ V505 Per } \\
\hline & & & present work & Marschall et al. (1997) \\
\hline Period (days) & $1.9009312 \pm 0.0000023$ & $2.9846514 \pm 0.0000037$ & $4.2220116 \pm 0.0000054$ & $4.22201924 \pm 0.00000004$ \\
\hline Epoch (HJD) & $2448500.1655 \pm 0.0030$ & $2448499.1545 \pm 0.0020$ & $2447808.5997 \pm 0.0006$ & $2447808.5998 \pm 0.0001$ \\
\hline $\mathrm{a}\left(R_{\odot}\right)$ & $8.75 \pm 0.06$ & $13.11 \pm 0.07$ & $15.059 \pm 0.079$ & $14.971 \pm 0.017$ \\
\hline $\mathrm{V}_{\gamma}\left(\mathrm{km} \mathrm{sec}^{-1}\right)$ & $21.91 \pm 0.45$ & $8.47 \pm 0.46$ & $-0.41 \pm 0.39$ & $+0.040 \pm 0.074$ \\
\hline $\mathrm{q}=\frac{m_{2}}{m_{1}}$ & $0.951 \pm 0.012$ & $0.983 \pm 0.011$ & $0.982 \pm 0.011$ & $0.985 \pm 0.002$ \\
\hline i (deg) & $78.8 \pm 1.02$ & $86.2 \pm 0.6$ & $88.18 \pm 0.11$ & $87.83 \pm 0.02$ \\
\hline $\mathrm{e}$ & 0.0 & 0.0 & 0.0 & 0.0 \\
\hline $\mathrm{T}_{1}(\mathrm{~K})$ & $6460 \pm 150$ & $8770 \pm 150$ & $6430 \pm 40$ & $6486 \pm 105$ \\
\hline $\mathrm{T}_{2}(\mathrm{~K})$ & $6204 \pm 180$ & $8683 \pm 180$ & $6414 \pm 60$ & $6383 \pm 132$ \\
\hline $\mathfrak{R}_{1}\left(\mathrm{R}_{\odot}\right)$ & $1.64 \pm 0.16$ & $2.19 \pm 0.08$ & $1.40 \pm 0.02$ & $1.29 \pm 0.03$ \\
\hline $\mathfrak{R}_{2}\left(\mathrm{R}_{\odot}\right)$ & $1.01 \pm 0.25$ & $1.37 \pm 0.05$ & $1.14 \pm 0.03$ & $1.27 \pm 0.07$ \\
\hline$M_{1}\left(\mathrm{M}_{\odot}\right)$ & $1.28 \pm 0.03$ & $1.72 \pm 0.03$ & $1.30 \pm 0.02$ & $1.265 \pm 0.006$ \\
\hline$M_{2}\left(\mathrm{M}_{\odot}\right)$ & $1.22 \pm 0.03$ & $1.69 \pm 0.03$ & $1.28 \pm 0.02$ & $1.247 \pm 0.006$ \\
\hline $\mathbf{M}_{b o l, 1}$ & $3.24 \pm 0.18$ & $1.27 \pm 0.11$ & $3.60 \pm 0.04$ & $3.58 \pm 0.07$ \\
\hline $\mathbf{M}_{b o l, 2}$ & $4.54 \pm 0.44$ & $2.33 \pm 0.12$ & $4.04 \pm 0.05$ & $3.69 \pm 0.09$ \\
\hline $\log g_{1}$ & $4.12 \pm 0.05$ & $3.99 \pm 0.04$ & $4.26 \pm 0.01$ & $4.32 \pm 0.02$ \\
\hline $\log g_{2}$ & $4.55 \pm 0.12$ & $4.39 \pm 0.04$ & $4.43 \pm 0.01$ & $4.33 \pm 0.02$ \\
\hline$\sigma_{R V, 1,2}\left(\mathrm{~km} \mathrm{sec}^{-1}\right)$ & $3.6,4.0$ & $4.1,4.4$ & $1.5,2.5$ & \\
\hline$\sigma_{B_{T}}(\mathrm{mag})$ & 0.12 & 0.10 & 0.09 & \\
\hline$\sigma_{V_{T}}(\mathrm{mag})$ & 0.10 & 0.09 & 0.10 & \\
\hline$\sigma_{H_{P}}(\mathrm{mag})$ & 0.017 & 0.015 & 0.009 & \\
\hline
\end{tabular}

determination of the stellar radii, which is strongly dependent on the branches of the minima in the lightcurve. The Marshall et al. lightcurve has eclipses mapped by about 400 points of excellent quality per band, while only 8 Hipparcos $H_{\mathrm{P}}$ and 18 Tycho $B_{\mathrm{T}}, V_{\mathrm{T}}$ measurements cover the eclipses. Even if the formal error of the solution is smaller in our case, this is no doubt the result of the very small number statistics. The difference in the radii between our and Marshall et al. solutions is $8.5 \%$ for the primary and $11 \%$ for the secondary. Looking at the lightcurve in Fig. 3 not a single $H_{\mathrm{P}}$ observation falls on the rising branch of the primary eclipse. No wonder then that a difference of the order of $10 \%$ exist in the radius determinations! Just doubling the number of Hipparcos observations (and thus statistically doubling the eclipse coverage) would have resulted in a determination of absolute radii of the components of V505 Per accurate to 3-4\%.

\subsection{Pegasi}

OO Peg is similar to V505 Per (well detached system, similar period, equal depth eclipses), but observationally more difficult: it is fainter (by $1.4 \mathrm{mag}$ ) and its early-A spectral type makes the Ca II lines weaker and the spectra dominated by broad Paschen lines. In fact, mean standard errors in Table 2 for photometry and radial velocities are twice those of V505 Per.

Contrary to V505 Per and V570 Per, we intentionally did not pay attention to the orbital phase when we observed $\mathrm{OO} \mathrm{Peg}$, in the attempt to simulate odd phase coverage by GAIA. This choice generated the quite uneven distribution of $R V$ points in Fig. 4, with just a single observation around the first quadrature.

In spite of such less-favorable photometric and spectroscopic observing conditions, the solution for OO Peg in Table 4 looks interesting anyway. The formal error in the semi-major axis is $0.5 \%$ (0.5\% for V505 Per), in the temperature of the secondary $2 \%(0.9 \%)$, in masses of both components $1.8 \%(1.5 \%)$, in the mass ratio $1.1 \%(1.0 \%)$, in systemic velocity $0.5 \mathrm{~km} \mathrm{~s}^{-1}\left(0.4 \mathrm{~km} \mathrm{~s}^{-1}\right), 4 \%$ for $R_{1}$ (vs. $1 \%$ for V505 Per), $4 \%$ for $R_{2}$ (vs. $3 \%$ ), and 0.6 degrees for $i$ (vs. 0.1 degrees).

\subsection{V570 Persei}

With V570 Per we explored a system very similar to V505 Per, but with twice the errors (cf. Table 2), affecting both photometry and spectroscopy and showing much less pronounced eclipses. Indeed, they are visible only in the $H_{\mathrm{P}}$ lightcurve (cf. Fig. 5). As for V505 Per, we planned the spectroscopic observations to obtain a good coverage at all orbital phases.

The system parameters mainly linked to radial velocities are well enough constrained: the formal error of the semi-major axis is $0.7 \%$ ( $0.5 \%$ for V505 Per), of masses of both components $2.4 \%(1.5 \%)$, of mass ratio $1.1 \%$ $(1.0 \%)$, of systemic velocity $0.45 \mathrm{~km} \mathrm{~s}^{-1}\left(0.4 \mathrm{~km} \mathrm{~s}^{-1}\right)$. Determination of radii and inclination obviously suffer from poorer sampling and larger noise of photometric data: the error of the radius of the primary is $10 \%$ 


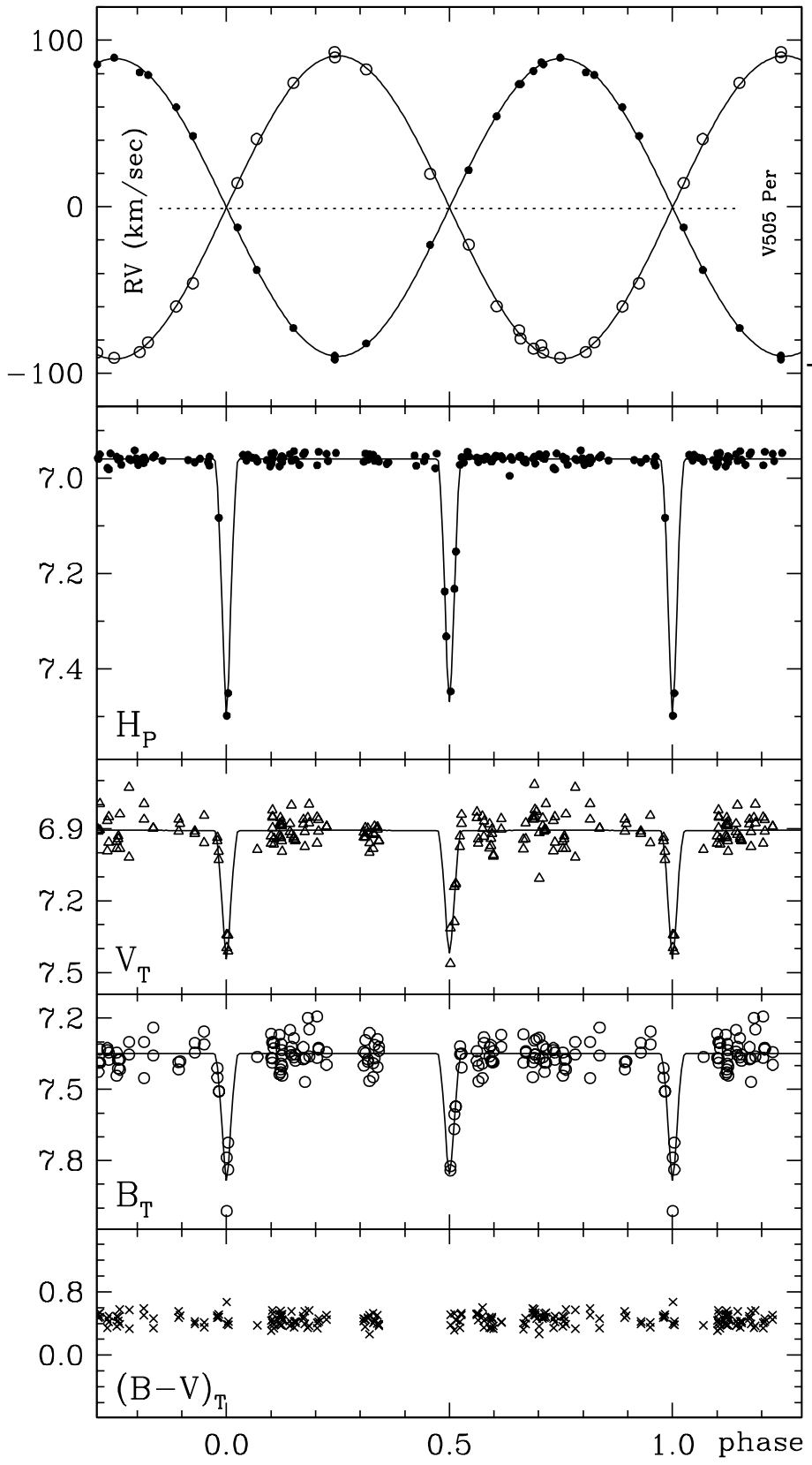

Fig. 3. Hipparcos $H_{\mathrm{P}}$ and Tycho $V_{\mathrm{T}}, B_{\mathrm{T}},(B-V)_{\mathrm{T}}$ lightcurves of V505 Per folded onto the period $P=4.2220116$ days. The lines represent the solution given in Table 4 .

for V570 Per (1\% for V505 Per) and for the secondary $25 \%(3 \%)$, while for inclination the error is 1.0 degrees (0.1 degrees). The temperature of the secondary star is constrained to a precision of 3\% (0.9\% for V505 Per).

\section{Discussion}

A detailed discussion of expected GAIA performances will necessarily be postponed to the end of the present series, when enough stars will be investigated to cover the span of spectral types and kinds of interaction (detached, semidetached, contact). This will provide us with a representative sample of the huge zoo of eclipsing binary stars

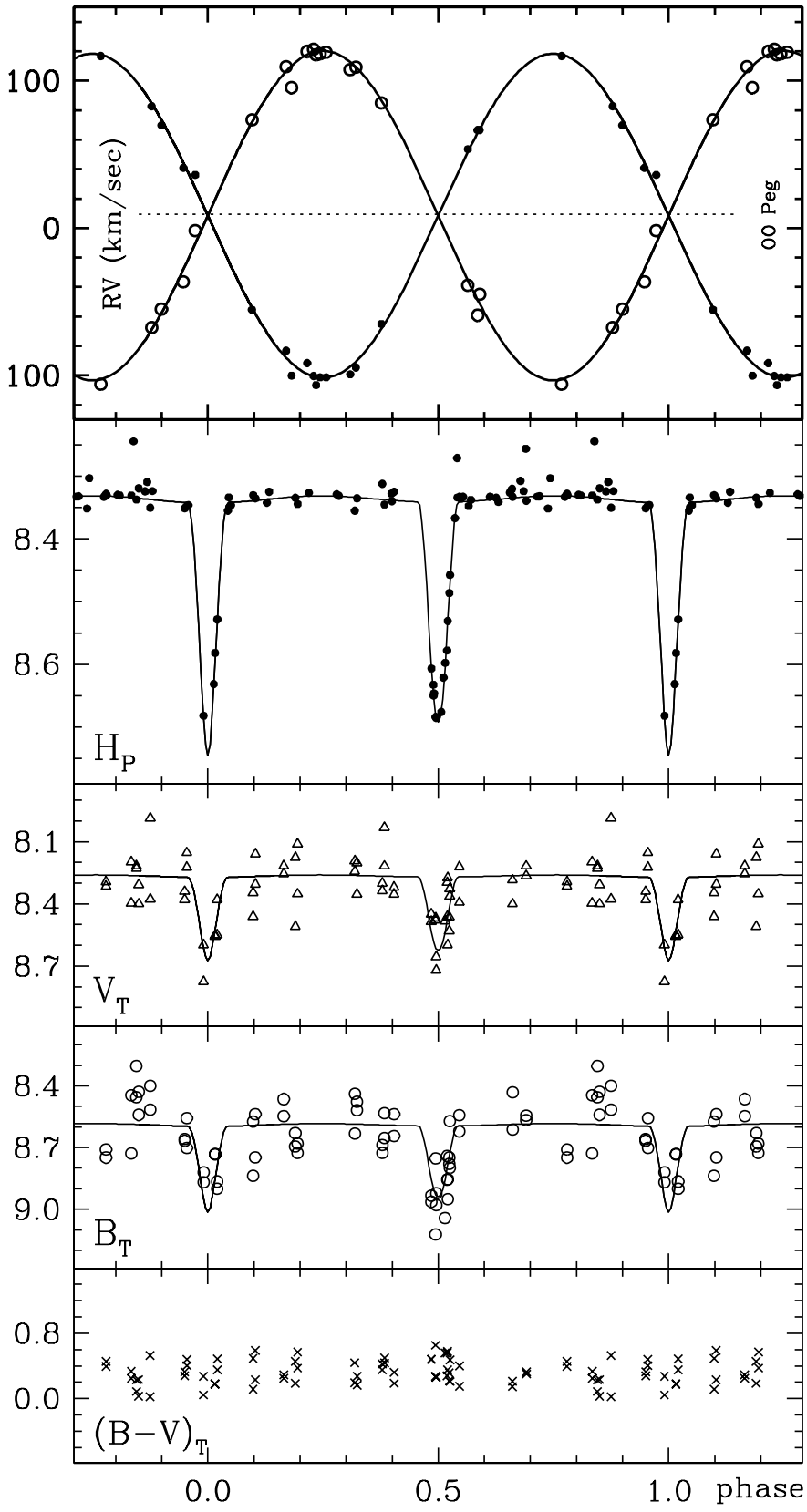

Fig. 4. Hipparcos $H_{\mathrm{P}}$ and Tycho $V_{\mathrm{T}}, B_{\mathrm{T}},(B-V)_{\mathrm{T}}$ lightcurves of OO Peg folded onto a period $P=2.9846514$ days. The lines represent the solution given in Table 4 .

within the observational capabilities of the coming ESA Cornerstone 6 mission.

Nevertheless, a few brief statements are in order to comment on the promising results on well detached, double-lined eclipsing binaries of A, F spectral types of a few days orbital period obtained here.

\subsection{Masses, orbital separations, baricentric velocities}

In this paper we used a mean of 23 spectra per star, spread over a wide range of $S / N$ (cf. Table 2). Orbital separations and individual masses are constrained to $\sim 0.7 \%$ and 


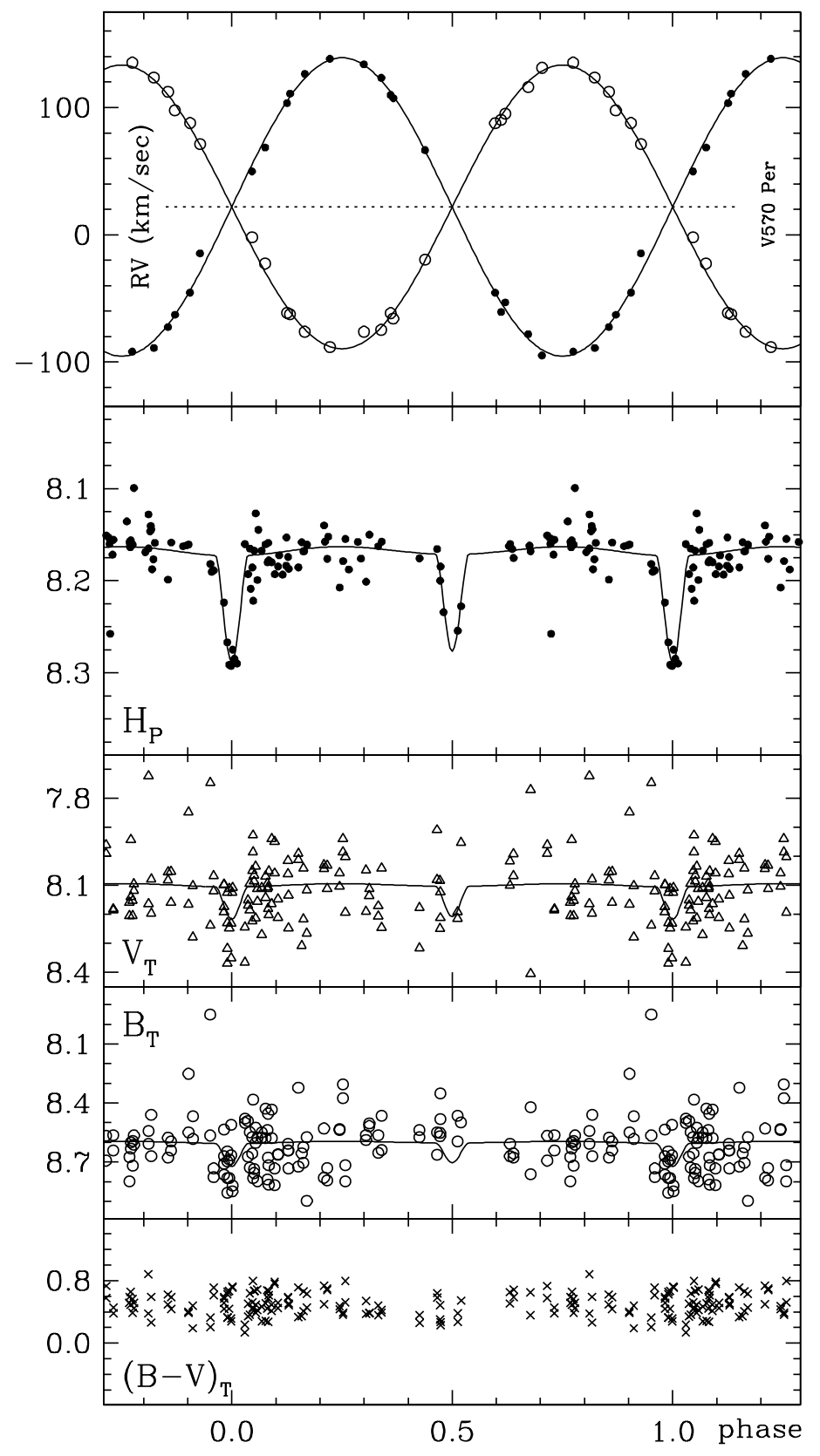

Fig. 5. Hipparcos $H_{\mathrm{P}}$ and Tycho $V_{\mathrm{T}}, B_{\mathrm{T}},(B-V)_{\mathrm{T}}$ lightcurves of V570 Per folded onto a period $P=1.9009312$ days. The lines represent the solution given in Table 4 .

$\sim 1.5 \%$, respectively. Thus the selected $\Delta \lambda=250 \AA$ wavelength range between 8490 and $8740 \AA$ is working fine for spectral types $\mathrm{A}$ and $\mathrm{F}$, even though it was optimized for cooler stars (the vast majority of field stars in the range of magnitude of interest to GAIA are G and $\mathrm{K}$ stars). GAIA will record the majority of its spectra at $S / N$ lower than obtained for this paper, but this will be compensated for by a much larger number of spectra per star $(\sim 67)$. It is therefore quite possible that a sizeable fraction of the welldetached, double-lined eclipsing binaries of A-F spectral type observed by GAIA will have their orbital separation and masses of both components determined to an accuracy of $\sim 1 \%$ provided that the spectral dispersion and
Table 5. Comparison between the Hipparcos distances and those derived from the parameters of the modeling solution in Table 4 (for OO Peg the range of possible distances according to Hipparcos are given).

\begin{tabular}{lccc}
\hline & \multicolumn{3}{c}{ distance (pc) } \\
\cline { 2 - 4 } & Hipparcos & this paper & Marshall et al. \\
V505 Per & $66 \pm 4$ & $59 \pm 4$ & $62 \pm 3$ \\
V570 Per & $117 \pm 14$ & $108 \pm 6$ & \\
OO Peg & $445_{840}^{304}$ & $295 \pm 17$ & \\
\hline
\end{tabular}

resolution will be not too far from $0.25 \AA /$ pix and $0.5 \AA$, respectively.

The baricentric velocities are constrained to better than $0.5 \mathrm{~km} \mathrm{~s}^{-1}$, which is fine in comparison to the accuracy of GAIA tangential motions, and also adequate to resolve the internal kinematics of stellar aggregates (clusters, associations, etc.)

\subsection{Period, epoch, eccentricity}

Periods, epochs and eccentricities can be derived to a high level of accuracy as this investigation has proved and the 5 years of planned mission lifetime for GAIA suggests.

For the program stars we adopted $e=0.0$ because modeling excluded any eccentricity larger than 0.01 , which can be taken as a rough indication of the accuracy to which eccentricity could be determined by GAIA observations.

Periods for the three program stars have been determined to such an accuracy that it would take $\Delta t \sim$ 170 years (on average) to bring the ephemeris out of phase for more than $10 \%$ of the orbital period. The 5 years of GAIA operation (compared to the time span of 3.36 years for Hipparcos data or 1.38 years for the spectral data used here), should expand the $10 \%$ accuracy horizon of the ephemeris to $\Delta t \sim 250$ years.

\subsection{Inclination, radii}

These parameters severely depend upon the way photometry maps the eclipse phases.

Hipparcos has collected about 110 measurements per star. If eclipses last for $1 / 10$ or fewer of the orbital period, one has to expect 10 or fewer photometric points to be distributed over the eclipse phases.

The situation will be more favourable with GAIA, having on average $1005=67 \times 15$ photometric observations grouped into $201=67 \times 3$ independent epochs.

\subsection{Temperature, surface gravity}

These two parameters are also dependent on GAIA photometry. However, they can be determined in a completely independent way.

Effective temperatures and surface gravities are in fact two of the basic outputs of a spectral synthesis analysis 
(e.g. based on Kurucz's or equivalent model atmospheres). The $0.25 \AA /$ pix dispersion and $0.5 \AA$ resolution GAIA spectra used here are adequate to derive temperatures to $\pm 30 \mathrm{~K}$ and gravities to 0.15 dex (cf. Munari \& Castelli 2000). The analysis of GAIA spectra of eclipsing binaries (mainly those obtained at quadratures when the spectra of both components are better observed) would therefore provide these quantities directly. The effective temperature and surface gravity determined directly from GAIA spectra could then be used to check a posteriori the results of modeling or used as inputs in the modeling process to decrease the number of parameters to be adjusted.

It is also worth mentioning that surface gravities will also be a by-product of GAIA astrometry, when combining parallaxes with multi-band photometry and spectral classification.

\subsection{Comparison with Hipparcos distances}

A natural check of the goodness of the orbital solution and modeling is to compare the distance to the program stars computed from the modeling parameters in Table 4 with the trigonometric parallaxes measured by Hipparcos. This is done in Table 5, together with the distance to V505 Per from the modeling of Marshall et al. (1997).

The high expected performances of GAIA observations are confirmed: ( $a$ ) the modeling of photometric lightcurves and radial velocity curves for the three program stars constrains the distance to a better accuracy than Hipparcos trigonometric parallax, and $(b)$ modeling distances and Hipparcos parallaxes agree inside the respective errors.

As for the effective temperature and surface gravity, trigonometric parallaxes determined directly by GAIA (at an average $200 \times$ better precision than Hipparcos) could then be used to check a posteriori the results of modeling or used as an input value in the modeling process itself.

\subsection{Prospects for GAIA}

The unique characteristic of GAIA as a single mission which combines astrometry, spectroscopy and photometry appears to offer special advantages compared to the classical ground-based approach to eclipsing binaries. The possibility of using effective temperatures, surface gravities and trigonometric parallaxes as fixed input values to the modeling of lightcurves and radial velocities should allow more confident derivation of other parameters, both stimulating a new modeling approach to eclipsing binaries and also granting lower errors to the solution.

By probing all constituents of the Galaxy (halo, disk, bulge) and detecting eclipsing binaries there, GAIA can foster an epochal leap in our knowledge of the basic stellar parameters and how they vary with mass, age and chemical abundance. This will be true not only on statistical grounds (given the huge number of observable eclipsing systems) but also for an object by object approach. This paper has in fact shown that an accuracy of $1 \%$ in the derived stellar parameters can be achieved by GAIA on a fraction of the detached eclipsing binaries observed during the mission.

Acknowledgements. Generous allocation of observing time with the Asiago telescopes has been vital to the present project as it has been the financial support by the Osservatorio Astronomico di Padova and by the Italian Space Agency (via grants to CISAS, University of Padova). It is a pleasure to thank PierLuigi Bernacca for his support; Panos Niarchos for his careful reading and commenting upon the manuscript; Bob Wilson for making available his further improved Wilson-Devinney program and for advice on its usage; Walter Van Hamme, Dirk Terrell, and Chris Stagg for contributing to the effectiveness of the WD98 and wd98k93 packages. The financial support of the Italian Space Agency (to UM), of NSERC and University of Calgary Research Grant Committee (to EFM) and the Slovenian Ministry for Research and Technology (to TZ) are kindly acknowledged.

\section{References}

Andersen, J. 1991, A\&AR, 3, 91

Bessell, M. S. 2000, PASP, 112, 961

Carquillat, J. M., Nadal, R., Ginestet, N., \& Pedoussaut, A. 1982, A\&A, 115, 23

Castelli, F., \& Munari, U. 2001, A\&A, 366, 1003

Gilmore, G., Perryman, M., Lindegren, L., et al. 1998, Proc. SPIE Conf., 3350, 541

Hauck, B., \& Mermilliod, M. 1998, A\&AS, 129, 431

Kallrath, J., \& Milone, E. F. 1999, Eclipsing Binary Stars: Modeling and Analysis (Springer-Verlag)

Kallrath, J., Milone, E. F., Terrell, D., \& Young, A. T. 1998, ApJ, 508, 308

Marschall, L. A., Stefanik, R. P., Lacy, C. H., et al. 1997, AJ, 114,793

Mérat, P., Safa, F., Camus, J. P., Pace, O., \& Perryman, M. A. C. 1999, in Proceedings of the ESA Leiden Workshop on GAIA, 23-27 Nov. 1998, Baltic Astron., 8, 1

Munari, U. 1999a, in Proceedings of the ESA Leiden Workshop on GAIA, 23-27 Nov. 1998, Baltic Astron., 8, 73

Munari, U. 1999b, in Proceedings of the ESA Leiden Workshop on GAIA, 23-27 Nov. 1998, Baltic Astron., 8, 123

Munari, U., \& Castelli, F. 2000, A\&AS, 141, 141

Munari, U., \& Lattanzi, M. G. 1992, PASP, 104, 121

Munari, U., \& Tomasella, L. 1999a, A\&A, 343, 806

Munari, U., \& Tomasella, L. 1999b, A\&AS, 137, 521

Oblak, E., \& Kurpinska-Winiarska, M. 2000, IAU Symp. C 200 The Formation of Binary Stars, ed. B. Reipurth, \& H. Zinnecker, 138

Perryman, M. A. C., de Boer, K. S., Gilmore, G., et al. 2001, A\&A, 369, 339

Popper, D. M. 1980, ARA\&A, 18, 115

Wilson, R. E. 1998, Computing Binary Star Observables, Univ. of Florida Astronomy Dept. 